\title{
Synthesis and studies on structural and optical properties of zinc oxide and manganese-doped zinc oxide nanoparticles
}

\author{
S. Ajin Sundar ${ }^{1}$, N. Joseph John ${ }^{2}$ \\ ${ }^{1}$ Department of Physics, Government Arts College, Udhagamandalam, Tamilnadu, India. Pin - 643002 \\ ${ }^{2}$ Department of Physics, Sethupathy Government Arts College, Ramanathapuram, Tamilnadu, India. \\ Pin -623502 \\ njosephjohn@gmail.com
}

DOI 10.17586/2220-8054-2016-7-6-1024-1030

\begin{abstract}
Wet chemical techniques have been used to synthesize undoped and Mn-doped nanoparticles at room temperature. Highly stable pure and 5.0 weight $\%$ Mn-doped $\mathrm{ZnO}$ nanoparticles have been prepared. The morphologies, structures and optical properties of the as-prepared samples were characterized by X-ray powder diffraction, scanning electron microscopy, energy dispersive X-ray spectroscopy and UV-Vis spectra. The results clearly reveled that both the pure and doped samples had a wurtzite hexagonal phase. The SEM studies illustrated that grain size decreases with Mn doping, with average diameter $\sim 30 \mathrm{~nm}$, which is in good agreement with the average crystalline size calculated by Scherrer's formula. The strong absorption band in the UV region for the prepared samples can be attributed to the band edge absorption of the wurtzite hexagonal $\mathrm{ZnO}$.
\end{abstract}

Keywords: nanoparticles, X-ray techniques, structural, FTIR, SEM, EDX.

Received: 1 March 2016

Revised: 25 August 2016

\section{Introduction}

Due to its wide band gap $(\sim 3.37 \mathrm{eV})$ and large exciton binding energy $(\sim 60 \mathrm{meV}), \mathrm{ZnO}$ is of great interest for photonic applications and its optical properties have been studied in detail. In recent years, due to prediction of possible ferromagnetic properties in transition metal-doped $\mathrm{ZnO}$ with Curie temperatures (Tc) above room temperature, studies of transition metal doped $\mathrm{ZnO}$ have attracted much interest [1,2]. Multifunctional materials come on stage accompanied with magnetic properties in diluted magnetic semiconductors (DMS), in which nonmagnetic ions replace some of the magnetic and non-magnetic cations. The origin of ferromagnetic properties in diluted semiconductors have been theoretically and experimentally studied $[3,4]$.

Among DMS materials, ZnO-based DMS has remarkable features: (i) Direct wide band gap at room temperature, (ii) access to the large bulk and high-quality single crystals of $\mathrm{ZnO}$ and (iii) large exciton binding energy. The latter is useful for having lasers with high efficiencies at room temperature [5,6]. In addition, ZnO is useful in solar cells and sensors as it is transparent conductive and piezoelectric [7]. Pure ZnO nanostructures show weak optical features that result from point defects such as oxygen vacancy or interstitial $\mathrm{Zn}$; therefore, they cannot be used directly in the industry [7]. As a result, doping $\mathrm{ZnO}$ with a convenient element is a method for engineering optical and magnetic properties. Furthermore, in order to make optoelectronic devices, n-type and p-type states are needed. It is relatively difficult to achieve p-type doping and this limitation is considered an obstacle to using $\mathrm{ZnO}$ in these applications [8].

Additionally, $\mathrm{ZnO}$ is lower in cost and is environmental friendly as compared to other metal oxides [9]. Normally, $\mathrm{ZnO}$ is studied in nanoscale; this is probably due to the fact that the high surface area to mass ratio of nanoparticles could enhance the adsorption of organic pollutants on the surface of particles as compared to the bulk materials [10]. Thus, in order to enhance the optical, magnetic, and electrical properties of $\mathrm{ZnO}$, transition metaldoped $\mathrm{ZnO}$ nanoparticles have been introduced. The modification of $\mathrm{ZnO}$ nanoparticles by impurity incorporation leads to possible application in UV optoelectronic and spin electronics [11]. Among the transition elements, Mn shows the maximum magnetic behaviors with electron effective mass $\sim 0.3 m e$ ( $m e=$ free electron mass); a doping of $\mathrm{Mn}$ into the $\mathrm{ZnO}$ semiconductor host lattice may result in large injected spins and carrier which make it suitable to be applied as diluted magnetic semiconductor (DMSs) [12].

$\mathrm{ZnO}$ has penetrated far into industry, and is one of the critical building blocks in modern society. Zinc oxide is in all likelihood one of the most abundant forms of any known material. The properties of $\mathrm{ZnO}$ are strongly dependent on its structure, including the morphology, aspect ratio, size, orientation, and crystal density. Due to its potential application in many areas, such as optoelectronic devices, solar cells, chemical sensor, and photocatalyst hence, $\mathrm{ZnO}$ has attracted much attention from researchers and scientists to develop it for technological applications. 
The term "doped" is used to modify the optical or magnetic properties of the host by adding impurities ions which become incorporated into the host lattice. Doping with $3 d$ metals such as $\mathrm{Mn}, \mathrm{Ni}, \mathrm{Fe}, \mathrm{Co}$, and $\mathrm{Cr}$ will increase the surface area and reduce the particle size of $\mathrm{ZnO}$ nanoparticles [13]. Mn is preferred for the doping of $\mathrm{ZnO}$ because the $d$ electron of $\mathrm{Mn}$ at $t_{2 g}$ level can easily overlap with the ZnO's valence bond, as compared with other transition elements. Among nanomaterials of transition metal oxides, $\mathrm{ZnO}$ is considered as a significant material for multiple applications, including catalytic, optical, magnetic, electrical, electrochemical and biological uses. These properties makes $\mathrm{ZnO}$ as a multifunctional material that finds applications in solar cell, biosensors, field effect transistors, light emitting diodes, diluted and ferromagnetic materials for spintronics, photocatalysis and anti-bacterial activity [13]. There are various studies which have shown that Mn-doped semiconductors have influenced the physical, chemical, and structural properties of undoped $\mathrm{ZnO}$ nanoparticles. For example, the optical properties of undoped $\mathrm{ZnO}$ nanoparticles especially on the tuning of the band gap can be greatly improved at the nanoscale by optimum Mn doping content $[14,15]$.

It was observed that doping with $3 d$ metals reduced the $\mathrm{Eg}$ of semiconductors by forming interband-gap localized levels [16]. The charge-transfer transition between the $d$-electron of dopant $\left(\mathrm{t}_{2 g}\right.$ levels) and the conduction band (CB) or valence band (VB) was reported [17]. Conversely, the metal's $d$-orbitals are mixed with the CB and VB of the semiconductors [18]. This overlap is because wide VB or CB directly decreases Eg. Recently, there has been much attention focused on modifying $\mathrm{ZnO}$ by doping with transition metals, such as $\mathrm{Ag}$ [19], Ni [20], $\mathrm{Cu}$ [21], Co [22], $\mathrm{Cr}$ [23], Ti [24]. These studies demonstrated that the metals can change the Eg of ZnO, and that the dopants can alter the $\mathrm{ZnO}$ grain size. This also demonstrated the presence of the $d$-electron because the $\mathrm{t}_{2 g}$ of manganese $(\mathrm{Mn})$ is very close to the VB $[17,25]$. More recently, the synthesis of a Mn-doped ZnO nano-crystal (not powder) was reported by wet-chemical techniques [26]. Moreover, $\mathrm{ZnO}$ nanocrystalline powders doped with 1,3 and $5 \% \mathrm{Mn}$ were prepared using a sol-gel process [27].

Zinc oxide is a II-VI compound semiconductor. Its properties, such as wide bandgap, large excitation binding energy and low threshold power for optical pumping are considered to be the eminent features of $\mathrm{ZnO}$. Thus, $\mathrm{ZnO}$ is said to be an efficient phosphor [28-31]. Type II-VI semiconductors, as a class of materials, have been and still are the subject of much intensive investigation. The growth of semiconductor technology in the early 1950's highlighted the limitations of silicon and germanium, which perhaps the character and the magnitude of the forbidden energy gap were the most disadvantageous. At first the extension in the range of energy gaps was sought in these III-V semiconductors, where considerable success has been achieved with InSb and GaAs in the low and high energy gap areas respectively. GaAs is today probably the most developed and well understood compound in existence. Concurrently with the later developments in the III-V semiconductors, systematic studies were made for several of these compounds.

The results of these studies have revealed much about the general nature of the II-VI semiconductors and the feature of chemical stability of the higher energy gap materials at room temperature offers an immediate advantage over the unstable III-V phosphides. Type II-VI semiconductors, in their broadest sense, include compounds formed from elements of group II and group VI of the periodic table. Zinc oxide has a wide band gap large excitation energy of $60 \mathrm{meV}$, high chemical stability, good piezo-electric properties, nontoxicity and biocompatibility. To synthesize $\mathrm{ZnO}$ nanoparticles with transition metals, several different methods have been employed, such as wet chemical methods like sol-gel [32], co-precipitation [33], combustion [34] etc. These nanoparticles have been used in a wide range of applications, such as sensors, varistors, piezoelectric, transducers, surface acoustic wave devices, phosphors, transparent conducting oxides, optoelectronic devices, ferromagnetic devices, and heterogeneous photo catalysts [35-40]. In this paper we report the synthesis and characterization of $\mathrm{Mn}$ doped and undoped zinc oxide via wet chemical method as well as the effect of $5.0 \mathrm{wt} \% \mathrm{Mn}$ doping in $\mathrm{ZnO}$ on its structural, morphological and optical properties.

\section{Experimental}

\subsection{Materials Used}

Analytical Reagent (AR) grade Zinc acetate dehydrate, Sodium Dodecyl sulfate, Sodium hydroxide and manganese chloride were used for synthesis of pure and manganese doped zinc oxide nanoparticles. Reagents were purchased from Merck India and used as received without any further purification.

\subsection{Synthesis of $\mathrm{ZnO}$ Nanoparticles}

For the synthesis of pure zinc oxide nanoparticles $10.966 \mathrm{~g}$ of zinc acetate dihydrate was added to $250 \mathrm{ml}$ water with vigorous stirring until an homogeneous solution was obtained. Next, $10.974 \mathrm{~g}$ of $\mathrm{NaOH}$ was added to the solution under stirring and to this solution $14.4 \mathrm{~g}$ of sodium dodecyl sulfate was added under continuous 
stirring until a $\mathrm{pH}$ of 12 was reached. This solution was was dried in a hot air oven at $160{ }^{\circ} \mathrm{C}$ overnight. The material was then ground in a mortar and pestle and the powder thus obtained was washed several times using ethanol and deionized water. The product was then dried at $60{ }^{\circ} \mathrm{C}$ overnight and the obtained product was calcined at $500{ }^{\circ} \mathrm{C}$ for 4 hours.

\subsection{Synthesis of Mn-Doped ZnO Nanoparticles}

For the synthesis of 5.0 weight $\%$ manganese doped $\mathrm{ZnO}, 0.7916 \mathrm{~g}$ manganese chloride was added to $250 \mathrm{ml}$ of water under vigorous stirring and $10.966 \mathrm{~g}$ zinc acetate was then added. Next, $10.974 \mathrm{~g}$ of sodium hydroxide was added to the solution with $14.4 \mathrm{~g}$ sodium dodecyl sulfate added under continuous stirring till the $\mathrm{pH}$ value reached 12 and the solution was dried in a hot air oven at $160{ }^{\circ} \mathrm{C}$ overnight. Then the material was taken and ground using a mortar and pestle and the obtained powder was washed several times using ethanol and deionized water. The product was dried at $60{ }^{\circ} \mathrm{C}$ for 2 days and the obtained product was then calcined at $500{ }^{\circ} \mathrm{C}$ for 4 hours.

\subsection{Characterization Studies}

The pure and manganese doped Zinc oxide nanoparticles thus obtained were characterized by UV, FTIR, XRD, SEM and EDAX. The seven synthesized samples were characterized using UV-visible spectroscopy by dissolving $0.1 \mathrm{~g}$ of each sample in $100 \mathrm{ml}$ doubly distilled water. The absorption data was recorded as a function of wavelength using a UV-visible spectrophotometer model-2202, India. XRD (X-ray Powder Diffraction) is rapid analytical technique primarily used for phase identification of crystalline material. The average crystallite size was determined from XRD peaks using Scherer's formula. The field emission Scanning Electron Microscopy (SEM) is one of the most versatile and well known analytical techniques, offering advantages like high magnification, large depth of focus, great resolution. Electrons generated from an electron gun enter a surface of sample and generate many low energy secondary electrons. The intensity of these secondary electrons is governed by the surface topography of the same. An image of the sample surface is therefore constructed by measuring secondary electron intensity as a function of the position of the scanning primary electron beam. EDX analysis is a useful tool widely used for chemical analysis. The intensity of backscattered electrons generated by electron bombardment can be correlated to the atomic number of the element within the sampling volume. Hence, qualitative elemental information can be revealed. The characteristic X-rays emitted from the sample serve as fingerprints and give elemental information for the samples, including semi-quantitative analysis, quantitative analysis, line profiling and spatial distribution of elements.

\section{Results and Discussion}

\subsection{UV-Visible Studies}

Figure 1 shows the UV-Visible spectra for zinc oxide and $5.0 \mathrm{wt} \% \mathrm{Mn}-\mathrm{ZnO}$. These graphs show absorption peaks at $381.62 \mathrm{~nm}$ and $379 \mathrm{~nm}$ respectively. The strong absorption band in UV region for Zinc oxide and manganese doped zinc oxide can be attributed to the band edge absorption of wurtzite hexagonal $\mathrm{ZnO}$, blue shift relative to its bulk $380 \mathrm{~nm}$. The zinc oxide sample that is doped with $\mathrm{Mn}$ also shows the absorption band similar to zinc oxide. When $\mathrm{Mn}$ doped in $\mathrm{ZnO}$ nanoparticles, the position of the absorption spectra is shifted towards the lower wavelengths or known as blue-shifted which correlated to the change in the optical band gap value. Generally, blue-shifting in the bandgap was due to $\mathrm{Mn}$ doping in the $\mathrm{ZnO}$ nanoparticles with replacement of the $\mathrm{Zn}$ ions in the $\mathrm{ZnO}$ lattice by $\mathrm{Mn}$ ions. This indicates that the band gap of $\mathrm{ZnO}$ nanoparticles increases when doped with Mn. Furthermore, the blue-shifting of the band gap energy with increased Mn doping concentration can be defined as the separation in the energy between the top of the valence band and the unoccupied energy states in the conduction band [41].

\subsection{FTIR Analysis}

FTIR studies were performed in order to determine the presence of functional groups and chemical bonding, as well as to study the surface changes on the particles. These analyses can reveal the quality or consistency of sample. FTIR transmission spectra were obtained using a Bruker, Alpha T; Germany spectrometer from $500-4000 \mathrm{~cm}^{-1}$ using $2 \mathrm{~cm}^{-1}$ resolution. Various peaks corresponding to the main absorption bands can be seen from the FTIR spectrum on figure 2. The broad absorption bands at $3577 \mathrm{~cm}^{-1}$ and $3578 \mathrm{~cm}^{-1}$ represent the $\mathrm{O}-\mathrm{H}$ stretching of the hydroxyl group. The band around $3100 \mathrm{~cm}^{-1}$ represents the O-H stretching [42]. The absorption bands observed between 1456 and $1330 \mathrm{~cm}^{-1}$ correspond to the asymmetric and symmetric stretching of the carbonyl group $\mathrm{C}=\mathrm{O}$. The small band at 879.97 and $628.104 \mathrm{~cm}^{-1}$ clearly indicate $\mathrm{Zn}-\mathrm{O}$ stretching. From these studies, the stretching mode of undoped $\mathrm{ZnO}$ is at $628.104 \mathrm{~cm}^{-1}$. When doped with $\mathrm{Mn}$, the absorption values were found to 


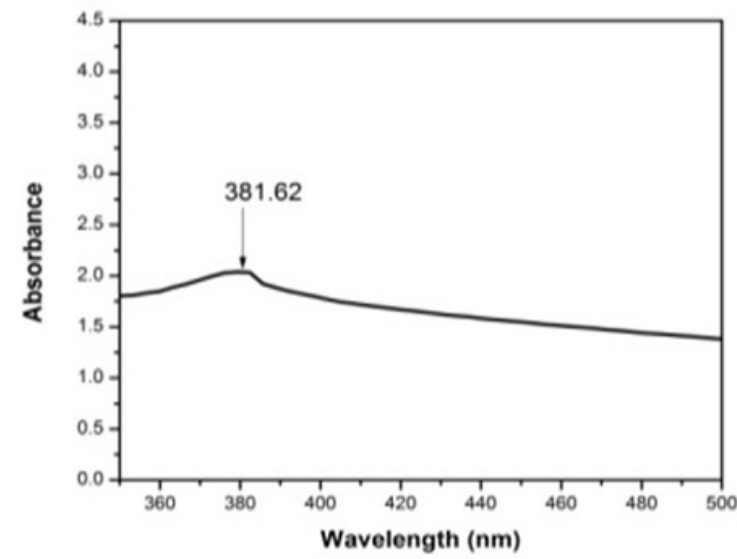

(a)

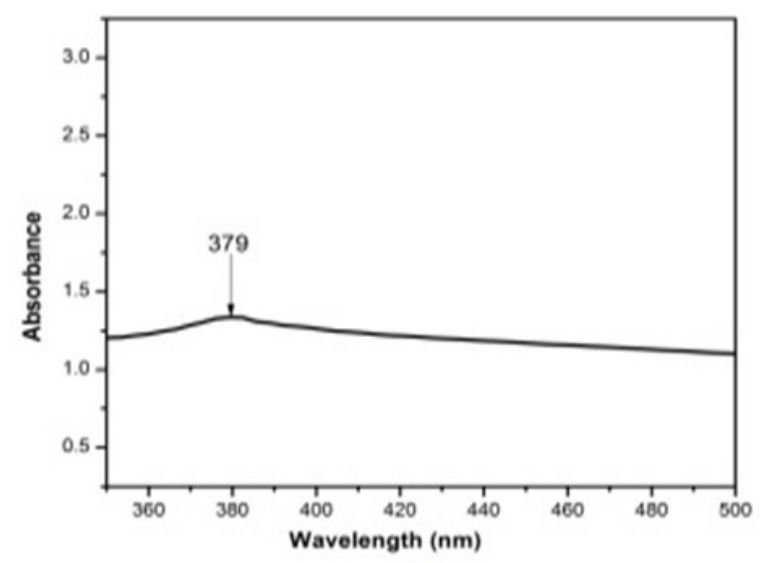

(b)

FIG. 1. UV spectra of (a) pure and (b) Mn-doped $\mathrm{ZnO}$

be blue-shifted at 618.8 and $595.30 \mathrm{~cm}^{-1}$ and were due to the Mn-O stretching and bending. Undoubtedly, this proves that the $\mathrm{Zn}-\mathrm{O}-\mathrm{Zn}$ network was perturbed by the presence of $\mathrm{Mn}$ in its environment as shown by the changes in the positions of the $\mathrm{ZnO}$ absorption bands [43].

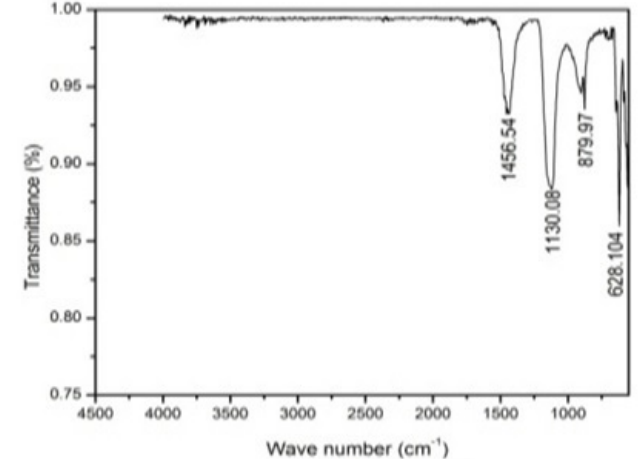

(a)

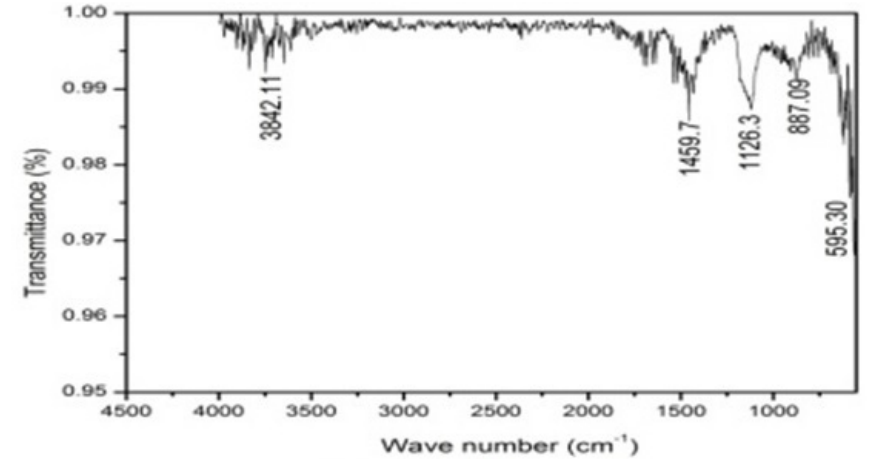

(b)

FIG. 2. FTIR Spectra (a) pure and (b) Mn doped $\mathrm{ZnO}$

\subsection{XRD Analysis}

Figure 3 illustrates $\mathrm{ZnO}$ and manganese doped $\mathrm{ZnO}$. (a) shows the XRD spectrum of $\mathrm{ZnO}$. The eleven major peaks were seen at 23.3, 25.3, 31.7, 34.6, 36.2, 47.6, 56.5, 62.9, 67.9, 68.0 and 69.8 which can be assigned to diffraction from (-112), (310), (100), (002), (101), (102), (110), (103), (200), (112) and (201) planes respectively, which is in good agreement with the standard value JCPDS no, 36-1451. (b) shows the XRD spectrum of Mn doped $\mathrm{ZnO}$. The twelve major peaks were seen at 23.3, 25.3, 31.7, 34.6, 36.2, 37.8, 47.6, 56.5, 62.9, 67.9, 68.0 and 69.8 which can be assigned to diffraction from $(-112),(310),(100),(002),(101),(521),(102),(110),(103)$, (200), (112) and (201) planes respectively. The sharp intense peaks of ZnO confirm the well-defined crystalline nature of $\mathrm{ZnO}$ and diffraction peak can be indicative of a hexagonal wurtize structured zinc oxide. The average crystallite size can be estimated using the Debye-Scherrer formula:

$$
2 d=\frac{0.9 \lambda}{\beta \cos \theta},
$$

where, $\beta$ is the full width at half maximum intensity [FWHM] corresponding to the diffraction angle $2 \theta$ in radian, and $\lambda$ is the wave length of $\mathrm{Cu}-\mathrm{k} \alpha$ radiation. The average crystallite size for the prepared nanocomposite was found to be $30.587 \mathrm{~nm}$ for pure sample and $28.510 \mathrm{~nm}$ for doped sample. 


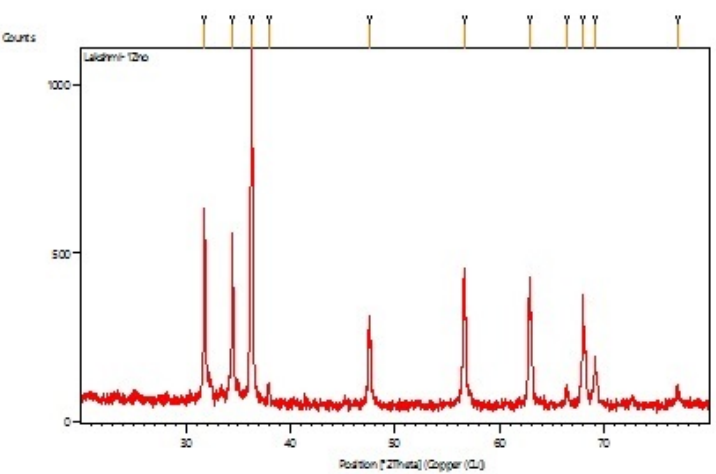

(a)

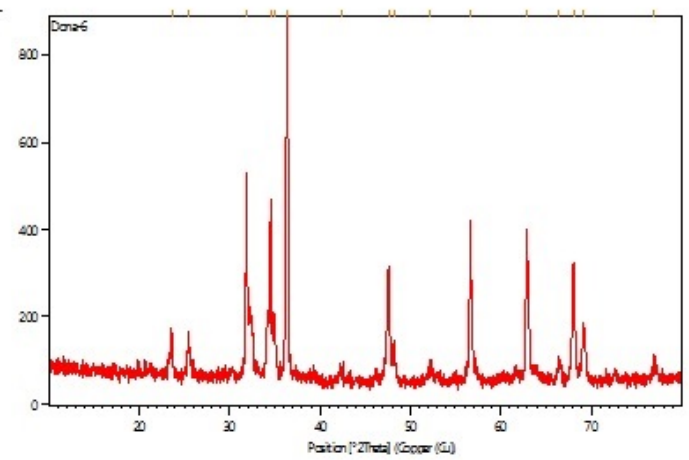

(b)

FIG. 3. XRD Pattern of (a) pure and (b) Mn doped $\mathrm{ZnO}$

\subsection{Particle Morphology}

Figure 4(a) shows the SEM image of undoped $\mathrm{ZnO}$ nanoparticles and the corresponding EDX is given in 5(a). The SEM image reveals that the particles are spherical in shape and mono dispersed with sizes less than $40 \mathrm{~nm}$. The SEM and EDX images of Mndoped ZnO are shown in 4(b) and 5(b) respectively. These clearly indicate the transformation of spherical rod shape with the particle size confinement with the result of Mndoping. In elemental analysis of zinc oxide, the peaks at $1.6 \mathrm{keV}, 8.6 \mathrm{keV}$ and $9.6 \mathrm{keV}$ confirm the presence of zinc and the peak at $0.53 \mathrm{keV}$ shows the presence of oxygen. The EDX spectra for the manganese-doped sample also confirmed the presence of zinc, oxygen and manganese. The peaks at $0.6 \mathrm{keV}$ and $5.9 \mathrm{keV}$ show the presence of manganese. The peak values at $1.6 \mathrm{keV}$ and $8.6 \mathrm{keV}$, confirm the presence of zinc and the peak at $0.53 \mathrm{keV}$ shows the presence of oxygen in the EDS spectra of manganese-doped $\mathrm{ZnO}$.

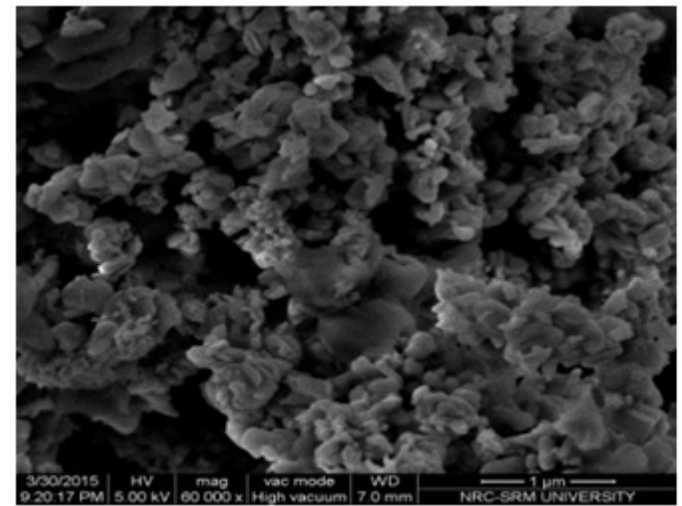

(a)

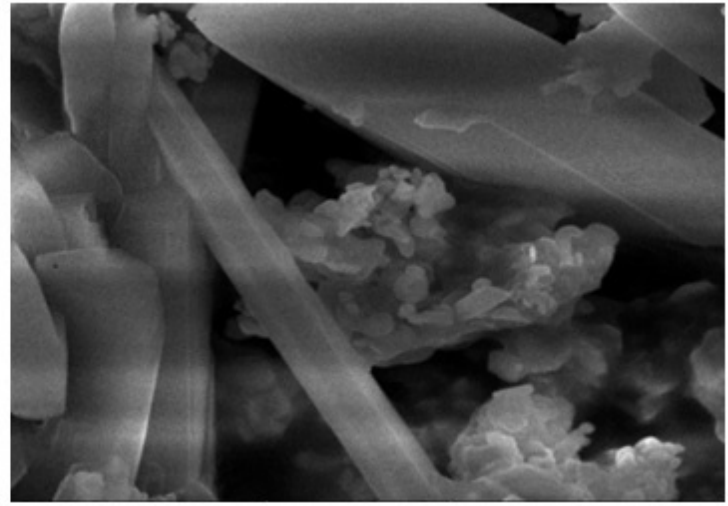

(b)

FIG. 4. SEM images of (a) pure and (b) Mn-doped $\mathrm{ZnO}$ nanoparticles

\section{Conclusion}

In summary, zinc oxide $(\mathrm{ZnO})$ and the transition metal manganese doped at various percentages of zinc oxide was synthesized by using zinc acetate dihydrate as a precursor. The characterization of the samples was done using FTIR, UV-Visible spectrophotometer, XRD, SEM and EDX. The presence of functional groups and chemical bonding was determined by FTIR. The absorption peaks for all the synthesized samples were determined by UVVisible spectrophotometry. The average crystallite sizes for all the samples were determined using XRD. The XRD peaks corresponded to the hexagonal wurtize structure. The SEM images were taken to determine the of the sample morphology. These analyses showed, the nanoparticles which were formed ranged from 75-90 nm. The EDX spectra were taken to determine the qualitative elemental information for zinc, oxygen and manganese in the synthesized particles. 


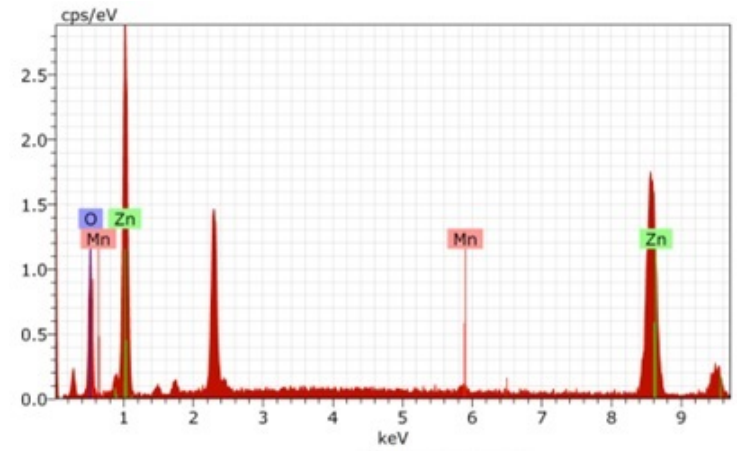

(a)

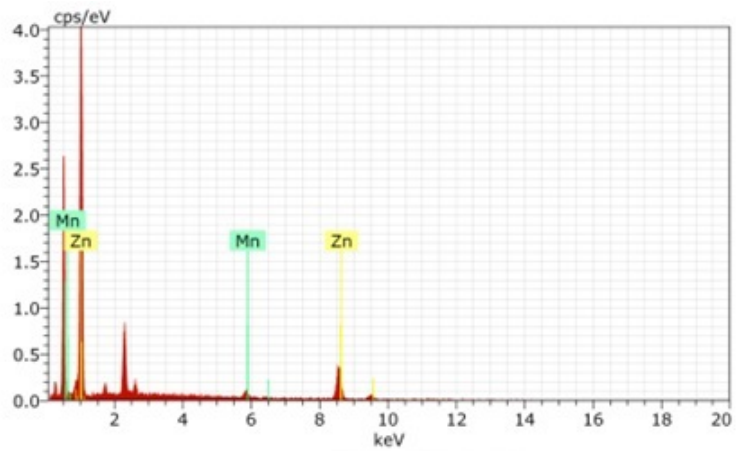

(b)

FIG. 5. EDS Spectra of (a) pure and (b) Mn-doped $\mathrm{ZnO}$

\section{Acknowledgement}

The author N. Joseph John acknowledges the University Grants Commission for providing financial support (No. F-MRP-4962/14 (MRP/UGC-SERO)).

\section{References}

[1] Dietl T., Ohno H., Matsukura F., Cibert J., and Ferrand D. Zener model description of ferromagnetism in zinc-blende magnetic semiconductors. Science, 2000, 287, P. 1019-1027.

[2] Sato K. and Katayama-Yoshida H. Low-dimensional Systems and Nanostructures. Physica E Amsterdam, 2001, 10, P. $251-258$.

[3] Pemmaraju C.D., Hanafin R., Archer T., Braun H.B., Sanvito S. Impurity-ion pair induced high-temperature ferromagnetism in co-doped ZnO. Phys. Rev. B, 2008, 78, P. 452-461.

[4] Sarsari I.A., Pemmaraju C.D., Salamati H., Sanvito S. Many-body quasiparticle spectrum of co-doped ZnO: A GW perspective. Phys. Rev. $B, 2013,87$, P. 781-790.

[5] Tsukazaki A., Ohtomo A., Onuma T., Ohtani M., Makino T., Sumiya M., Ohtani K., Chichibu S.F., Fuke S., Segawa Y., et al. Repeated temperature modulation epitaxy for p-type doping and light-emitting diode based on ZnO. Nat. Mater., 2004, 1, P. 42-46.

[6] Ozgur U., Alivov Y.I., Liu C., Teke A., Reshchikov M., Dogăn S., Avrutin V., Cho S.-J., Morkoc H. A comprehensive review of ZnO materials and devices. J. Appl. Phys., 2005, 98(4), P. 341-352.

[7] Ahmad M., Zhao J., Iqbal J., Miao W., Xie L., Mo R., Zhu J. Conductivity enhancement by slight indium doping in ZnO nanowires for optoelectronic applications. J. Phys. D: Appl. Phys., 2009, 42(16), P. 158-165.

[8] Teklemichael S.T., Oo W.H., McCluskey M., Walter E.D., Hoyt D.W. Acceptors in ZnO nanocrystals. Appl. Phys. Lett., 2011, 98(23), $232-422$.

[9] Iqbal J., Liu X., Majid A., and Yu R. Synthesis and physical properties of Mn doped ZnO dilute magnetic semiconductor nanostructures. Journal of superconductivity and Novel Magnetism, 2011, 24(1-2), P. 699-704.

[10] Raval H.D. and Gohil J.M. Nanotechnology in water treatment:an emerging trend. International Journal of Nuclear Desalination. 2010, 4(2), P. 184-188.

[11] Wang L.W., Xu Z., Zhang F.J., Zhao S.L., and Lu L.F. Structure, optical, and magnetic properties of Mn-doped ZnO films prepared by sputtering. International Journal of Minerals, Metallurgy and Materials, 2010, 17(4), 475-480.

[12] Shi-Wei W., Ming-Yuan Z., Min Z., et al. Effects of pulsed magneticfield onMn-doped ZnO diluted magnetic semiconductorprepared by hydrothermalmethod. Acta Physica Sinica, 2012, 61(19), P. 873-881.

[13] Yan X.X. and Xu G.Y. Effect of sintering atmosphere on the electrical and optical properties of (ZnO)1-x(MnO2)x NTCRceramics. Physica $B, 2009$, 404(16), P. 2377-2381.

[14] Abdollahi Y., Abdullah A., Zainal Z., and Yusof N. Synthesisand characterization of Manganese doped ZnO nanoparticles. International Journal of Basic and Applied Sciences, 2011, 4, P. 62-69.

[15] Soni B., Deshpande M.P., Bhatt S., Garg N., Pandya N., and Chaki S.H. Influence ofMn doping on optical properties of ZnOnanoparticles synthesized by microwave irradiation. Journal of Optics, 2013, 42(4), P. 328-334.

[16] Campet G., Jakani M., Doumerc J.P., Claverie J., Hagenmuller P. Photoconduction mechanisms in titanium and rare earth n-type semiconducting electrodes with pyrochlore and perovskite structures. Solid State Communications, 1982, 42(2), P. 93-6.

[17] Choi W., Termin A., Hoffmann M. The role of metal ion dopants in quantum-sized $\mathrm{TiO}_{2}$ : correlation between photoreactivity and charge carrier recombination dynamics. The Journal of Physical Chemistry, 1994, 98(51), P. 13669-13679.

[18] Umebayashi T., Yamaki T., Itoh H., Asai K. Analysis of electronic structures of 3d transition metal-doped TiO 2 based on band calculations. Journal of Physics and Chemistry of Solids, 2002, 63(10), P. 1909-1920.

[19] Wang R., Xin J.H., Yang Y., Liu H., Xu L., Hu J. The characteristics and photocatalytic activities of silver doped ZnO nanocrystallites. Applied Surface Science, 2004, 227(1-4), P. 312-317.

[20] Cong C.J., Hong J.H., Liu Q.Y., Liao L., Zhang K.L. Synthesis, structure and ferromagnetic properties of Ni-doped ZnO nanoparticles. Solid State Communications, 2006, 138(10-11), P. 511-515.

[21] Kanade K.G., Kale B.B., Baeg J.-O., Lee S.M., Lee C.W., Moon S.-J., Chang H. Self-assembled aligned Cu doped ZnO nanoparticles for photocatalytic hydrogen production under visible light irradiation. Materials Chemistry and Physics, 2007, 102(1), P. 98-104. 
[22] Volbers N., Zhou H., Knies C., Pfisterer D., Sann J., Hofmann D.M., Meyer B.K. Synthesis and characterization of ZnO:Co $2+$ nanoparticles. Applied Physics A: Materials Science \&amp. Processing, 2007, 88(1), P. 153-5.

[23] Li L., Wang W., Liu H., Liu X., Song Q., Ren S. First principles calculations of electronic band structure and optical properties of Cr-doped ZnO. The Journal of Physical Chemistry C, 2009, 113(19), P. 8460-8464.

[24] Samaele N., Amornpitoksuk P., Suwanboon S. Morphology and optical properties of ZnO particles modified by diblock copolymer. Materials Letters, 2010, 64(4), P. 500-502.

[25] Halperin B., Lax M. Impurity-band tails in the high-density limit. I. Minimum counting methods. Physical Review, 1966, 148(2), P. 722740.

[26] Ullah R., Dutta J. Photocatalytic degradation of organic dyes with manganese-doped ZnO nanoparticles. Journal of Hazardous materials, 2008, 156(1-3), P. 194-200.

[27] Tsuzuki T., Smith Z., Parker A., He R., Wang X. Photocatalytic activity of manganese-doped ZnO nanocrystalline powders. Journal of the Australian Ceramic Society, 2009, 45(1), P. 58-62.

[28] Yang Z.K., Yu P., Wang G.K.L., Kawasaki M., Ohtomo A., Koinuma H. and Segawa Y. Ultraviolet Spontaneous and Stimulated Emission from ZnO Microcrystallites at Room Temperature. Solid State Commun., 1997, 103, P. 459-461.

[29] Bagnall D.M., Chen Y.F., Zhu Z., Yao T., Koyama S., Shen M.Y. and Goto G. Optically pumped lasing of ZnO at room temperature. Appl. Phys. Lett., 1997, 70, P. 2230-2232.

[30] Kawasaki M., Ohtomo A., Ohkubo I., Koinuma H., Tang Z.K., Yu P., Wong G.K.L., Zhang B.P. and Segawa Y. Room temperature ultraviolet emission fromself assembled ZnOmicrocrystalline thin films. Mater. Sci., 1998, B56, P. 239.

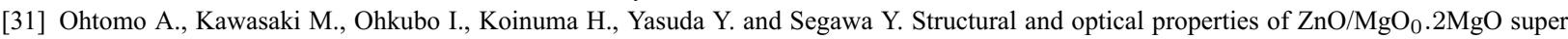
lattics. Appl. Phys. Lett., 1999, 75, P. 980.

[32] Brus L.E., Harkless J.A.W., and Stillinger F.H. Theoretical Metastability of Semiconductor Crystallites in High Pressure Phases, with Application to Beta-Tin Structure Silicon. J. Am. Chem. Soc., 1996, 118, P. 4834-4840.

[33] Abrishami M.E., et. al. Structural and optical properties of zinc oxide nano powders doped with Mn. Phys. Status Solidi C, 2010, 7 , P. 1595-1601.

[34] Yadav R.S., Pandey A.C., and Sanjaya S.S. Chalcogenide Lett., 2009, 6, P. 233-240.

[35] Riahi-Noori N., et. al. Optical properties of europium doped bunches of $\mathrm{ZnO}$ nanowires synthesized by co precipition method. J. Ceram. Process. Res., 2008, 9,P. 246-252.

[36] He J.H., Hsin C.L., Liu J., Chen L.J. and Wang Z.L. Piezoelectric Gated Diode of a Single ZnO Nanowire. Adv. Mater., 2007, 19, P. 781-788.

[37] Lee C.V., Haung Y.T., Su W.F., Lin C.F. Electroluminescence from ZnO nanoparticles/ organic nanocomposites. Appl. Phys. Lett., 2006, 89(23), P. 11-16.

[38] Garcia M.A., et. al. Magnetic properties of ZnO nanoparticles. Nano Lett., 2007, 7, P. 1489.

[39] Fonoberov V.A. and Balandin A.A. J. Nanoelectron Optoe. 2006, 119, P. 78-86.

[40] Kwon S.S., et. al. ZnO quantum dots: Physical properties and optoelectronic applications. Adv. Mater., 2008 ,20, P. $4557-4565$.

[41] Visvanatha R., Sapra S., Gupta S.S., et. al. Synthesis and characterization of Mn doped ZnO nanocrystals. Journal of Physical Chemistry $B, 2004,108(20)$, P. 6303-6310.

[42] Hao M.Y., Lou S.Y., Zhou S.M., Yuan R.J., Zhu G.Y. and Li N. Structural, optical, and magnetic studies of manganese doped zinc oxide hierarchical microspheres by self assembly of nanoparticles. Nanoscale Research Letters, 2012,7(100), P. 111-113.

[43] Wu D., Huang Z., Yin G., et. al. Preparation structure and properties of Mn-doped ZnO rod arrays. CrystEngComm, 2010, 12(1), P. $192-198$. 